\title{
Asertividad en practicantes de psicología de una institución colombiana de educación superior ${ }^{\star}$
}

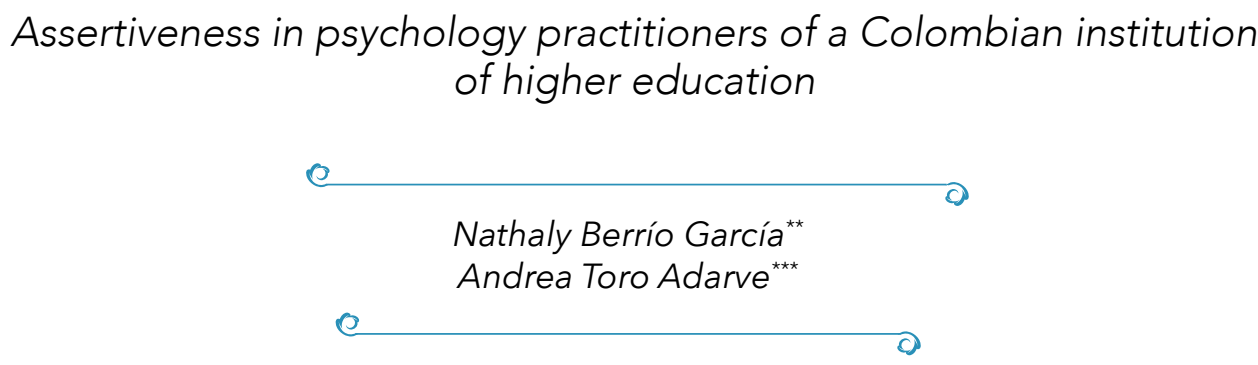

Recibido 08. 10.2018 • Arbitrado 29. 10. 2018 •

Aprobado 15.11. 2018

Articulo derivado de la investigación realizada para otra el título de psicólogo, Corporación Universitaria Minuto de Dios.

** Psicóloga, Magíster en Psicoterapia, Candidata a Doctora en Epidemiología y Bioestadística. Docente Investigadora Corporación Universitaria Minuto de Dios, nberriogarc@uniminuto.edu.co, ORCID: 0000-0002-3574-4101,

*** Psicóloga, Corporación Universitaria Minuto de Dios, atoroadarve@ uniminuto.edu.co, ORCID : 00000003-2583-2877

\section{Resumen}

El objetivo del estudio fue describir los niveles de auto y heteroasertividad en universitarios según las variables sociodemográficas sexo, edad, campo de práctica, nivel de práctica y semestre en curso. Se aplicó el cuestionario ADCA-1 en 113 practicantes de psicología. Los resultados mostraron que los estudiantes obtuvieron puntajes altos en ambas variables dependientes. El promedio de los evaluados en la variable heteroasertividad fue mayor que en autoasertividad. Se discuten las conclusiones, y se brindan recomendaciones para investigaciones futuras.

Palabras clave: asertividad, autoasertividad, heteroasertividad, universitarios, practicantes de psicología.

\section{Abstract}

The aim of the study was to describe the levels of auto and heteroassertiveness in university students according to sociodemographic variables sex, age, field of practice, level of practice and semester in progress. The ADCA-1 questionnaire was applied in 113 psychology practitioners. 
Findings revealed high scores in both assertiveness and hetero assertiveness but hetero assertiveness was higher than auto assertiveness. Conclusions and recommendations are discussed for future investigations.

Keywords: assertiveness, auto assertiveness, heteroassertiveness, university students, psychology practitioners.

\section{Introducción}

La asertividad no ha sido concebida como un constructo único, sino como un compendio de habilidades sociales. Dichas habilidades son conceptualizadas como un conjunto de conductas emitidas en contextos interpersonales que posibilitan la expresión de "sentimientos, actitudes, deseos, opiniones o derechos de un modo adecuado a la situación, respetando esas conductas en los demás y que, generalmente resuelve los problemas inmediatos de la situación mientras minimiza la probabilidad de futuros problemas" (Caballo, 1986, p. 6).

Las habilidades sociales permiten desenvolverse correctamente en las relaciones interpersonales. Son conductas aprendidas que tienen como fin responder a las demandas de la sociedad de manera efectiva, y también son medios para lograr los objetivos de un individuo. Las habilidades sociales se caracterizan porque se adaptan al contexto y brindan la posibilidad de entender el punto de vista de otra persona, es decir, permiten el intercambio social satisfactorio y eficaz (Merino, 2010).

Además, las habilidades sociales implican tres dimensiones del individuo: la conductual, que evidencia lo que emerge de la conducta al relacionarse con el otro, sus gestos, tono de voz, postura, etc.; la cognitiva, que engloba todo lo relacionado con creencias, pensamientos, percepciones, es decir, lo no tangible; y, por último, la dimensión fisiológica, en la que están las variables de orden somático (respiración, velocidad del pulso, sudoración, movimientos repetitivos, etc.) (Merino, 2010).

Por otra parte, García y Magaz (2000) definen las habilidades sociales como respuestas que tienen como fin empezar, conservar y terminar relaciones sociales que representan un agrado y /o beneficio para la persona misma y para el otro.

Asimismo, las habilidades sociales se clasifican en dos categorías: las habilidades sociales directas que incluyen respuestas influyentes claramente tanto en el ambiente como en las respuestas del receptor, pudiendo tener 
consecuencias positivas o negativas en la relación y en la persona; y las indirectas, que son las conductas subjetivas y no observables (cogniciones), que influyen en el ambiente y en la relación interpersonal con un individuo (García \& Magaz, 2000).

Adicionalmente, los individuos "habilidosos socialmente" tienen la capacidad de relacionarse con los demás de forma óptima, entablan una conversación, crean una relación con facilidad, y en la mayoría de las situaciones, las personas con las que interactúan terminan con una sensación agradable al finalizar la interacción (Kelly, 2002).

Acorde con lo anterior, las habilidades sociales son primordiales, ya que los humanos son sujetos sociales y al formar relaciones interpersonales se genera y aumenta el bienestar, la calidad de vida, la armonía, la autoestima y la salud mental en general. Y en oposición, el déficit de estas habilidades sociales genera malestar, estrés y tensión en las personas. En ese orden de ideas, las personas con pocas habilidades sociales podrían presentar altos índices de depresión, ansiedad, rechazos, frustraciones y demás consecuencias psicosomáticas (Roca, 2003).

También, se ha establecido que las habilidades sociales se evidencian en las respuestas frente a situaciones específicas en las cuales un individuo expresa sus deseos, creencias, necesidades, sentimientos, preferencias, etc., de manera asertiva y sin ansiedad. Comunicar de manera efectiva lo anteriormente mencionado repercute en refuerzos positivos externos para el individuo (Gismero, 2000).

En consecuencia, la asertividad como conjunto de habilidades sociales, es un constructo que aún no tiene una definición universal, debido a su amplitud conceptual (Caballo, 1983). La asertividad es definida como una habilidad personal que hace que las personas sean directas y honestas, ya que pueden expresar sus sentimientos, opiniones y pensamientos de forma oportuna y adecuada, sin confrontar las creencias y derechos del otro (Llacuna \& Pujol, 2004).

Por lo tanto, para la asertividad se establecen dos subconstructos: heteroasertividad y autoasertividad. La heteroasertividad es definida operacionalmente "como la clase de comportamiento que constituye un acto de respeto a la expresión sincera y cordial de los sentimientos y de valores, gustos, deseos o preferencias de los demás" (García \& Magaz, 2000, p.14.). En contraste, la autoasertividad es "la clase de comportamiento que constituye un acto de expresión sincera y cordial de los sentimientos propios y de defensa de los propios valores, gustos, deseos o preferencias" (García \& Magaz, 2000, p.14.). 
En síntesis, la autoasertividad hace referencia a la defensa de los derechos individuales y la heteroasertividad a la defensa de los derechos de los otros. No obstante, teniendo en cuenta que los seres humanos se caracterizan por la subjetividad, y que en ninguna medida dos humanos son totalmente iguales, tienen las mismas habilidades, etc., García y Magaz (2000) crearon cuatro categorías que pretenden clasificar los estilos habituales de interacción de las personas: pasivo (nivel elevado de heteroasertividad y escasa autoasertividad), agresivo (alta autoasertividad y mínima heteroasertividad), asertivo (estilo ideal que evidencia un equilibrio entre los dos subconstructos, mismo nivel de autoasertividad que de heteroasertividad), y pasivo-agresivo (nivel mínimo tanto de autoasertividad como de heteroasertividad). Las personas asertivas deberían emitir conductas que conlleven las dos perspectivas de la asertividad.

En ese orden de ideas, para una correcta realización de la práctica profesional, los estudiantes de psicología inscritos en esta asignatura deberán evidenciar habilidades sociales que les permitan comprender, explicar y solucionar las diversas situaciones complejas que se presenten en su ejercicio de práctica profesional, con el fin de reducir sustancialmente el riesgo de que dichas situaciones se vuelvan a presentar.

De ahí que, el Centro Progresa (2017) elaborara un perfil que refiere esas capacidades, habilidades y aptitudes que debe tener un estudiante en los diferentes campos de acción de la Psicología y, a su vez, las funciones que debe realizar en los mismos, como practicante profesional. En todos los ámbitos, educativo, social - comunitario, organizacional, jurídico y clínico, el estudiante debe realizar funciones como diagnosticar, realizar programas de promoción y prevención, intervenir individual y colectivamente, asesorar, etc. De modo que cada una de estas funciones requiere que los practicantes empleen una serie de habilidades, entre ellas, la asertividad.

Lo anterior se evidencia también desde la instancia que representa y regula el ejercicio de la Psicología en Colombia: el Colegio Colombiano de Psicólogos (Colpsic, 2014), el cual propone competencias por campos de la labor del Psicólogo, indicando que dichas competencias requieren que el profesional en Psicología ponga en acción competencias comunicativas, interpersonales, éticas, integrales, teóricas, investigativas, sociales etc. y entre esas, se hipotetiza que la asertividad es importante y necesaria para realizar de manera efectiva el ejercicio de la Psicología.

En relación con los antecedentes de investigación sobre asertividad se ha establecido que la falta de asertividad, relacionada con las patologías alimentarias, puede empeorar y acrecentar su sintomatología. Además, que la baja 
auto-aserción puede ser un factor para predecir un trastorno alimenticio (Behar, Manzo \& Casanova, 2005).

También, Londoño y Valencia (2008) se percataron que, a mayor ingesta de alcohol, los participantes de su investigación tenían menor resistencia a la presión social indirecta/directa y menor asertividad. De manera análoga, se compararon las características asertivas de dos grupos de estudiantes: Usuarios de drogas ilícitas y no usuarios de drogas, y se reportó que los "no usuarios" poseen estrategias asertivas de enfrentamiento más eficaces a los problemas, además, la autoeficacia, el control conductual y la aceptación de los demás tienen un nivel más alto en comparación al grupo consumidor (Velázquez, Arellanez \& Martínez, 2012).

Por otra parte, se ha confirmado que los alumnos con bajo desempeño en asertividad presentan niveles altos de agresividad y ansiedad y son también los que muestran menor rendimiento académico (González, Guevara, Jiménez \& Alcázar, 2018); y se ha evidenciado una tendencia a presentar un mayor nivel de asertividad, cuando hay mayor nivel de autoestima (León, Rodríguez, Ferrel \& Ceballos, 2009).

Asimismo, en relación con la edad y el sexo, Coppari et al. (2009) señalan que hay un mayor porcentaje de mujeres no asertivas que hombres, mientras que Torres (2015) sostiene que las mujeres son más asertivas que los hombres. Respecto a la edad, Cañón y Rodríguez (2011) concluyen que a medida que aumenta la edad de los evaluados aumenta la asertividad.

Adicionalmente, para las investigaciones de Gutiérrez y López (2015) y Rondón, Cardozo y Lacasella (2018) se aplicó el Autoinforme de Actitudes y Valores en las Interacciones Sociales (ADCAS). Los primeros se percataron de que los hombres obtuvieron mayor puntaje que las mujeres tanto en autoasertividad como en heteroasertividad. Los resultados de la segunda investigación sugieren que las variables depresión y comunicación asertiva se relacionan directamente, y que, a menor comunicación asertiva, mayor depresión.

Por tanto, el objetivo del estudio fue describir los niveles de auto y heteroasertividad en los practicantes de psicología de una Institución Colombiana de Educación Superior (IES), según las variables sociodemográficas sexo, edad, campo de práctica, nivel de práctica y semestre en curso.

Por ende, con base en la revisión detallada, y lo reportado a continuación, este estudio generó resultados que podrán emplearse posteriormente para diseñar programas de promoción y prevención, con el fin de fomentar y fortalecer habilidades sociales en los practicantes de psicología, en el campo de práctica. 
En relación con lo anterior, el estudio fue conveniente porque servirá a próximos investigadores para producir nuevo conocimiento y desarrollar nuevas investigaciones, y respecto a las áreas internas de la IES como la Dirección de Bienestar Institucional y Centro Progresa, los resultados son relevantes para el diseño de propuestas de talleres de inserción a los diferentes campos de práctica profesional, que enfaticen en la asertividad.

Por último, la investigación tiene relevancia social, ya que es necesario e importante que un psicólogo, en cualquier campo de acción, emplee bien las habilidades sociales para que sus intervenciones sean las adecuadas. A su vez, el fortalecimiento de estas habilidades permitirá que los practicantes de Psicología se desempeñen de la mejor manera posible en la realización de su ejercicio de práctica profesional.

\section{Método}

Diseño: Estudio no experimental, de tipo transversal y con alcance descriptivo. Las variables dependientes de heteroasertividad y autoasertividad se especificaron según las variables sociodemográficas como sexo, edad, ámbito de práctica, nivel de práctica y semestre académico.

Participantes: Se empleó una muestra de 113 estudiantes de una institución colombiana de educación superior. Los criterios de inclusión fueron que tuvieran matriculada la asignatura de práctica profesional en el 2018, y que estuvieran desarrollando actividades en una agencia de práctica. Se excluyeron los estudiantes que estaban matriculados, pero no tenían agencia de prácticas.

Se eligió una técnica de muestreo polietápica. En primer lugar, se filtró la base de datos de practicantes matriculados, eliminando unidades muestrales con dos o más prácticas matriculadas, para contabilizarlas una sola vez. Luego, se les envió un formulario virtual para consultar si tenían agencia de práctica y su campo de acción. De los 130 que contestaron el formulario, debían evaluarse 97 de acuerdo con el cálculo del tamaño de la muestra para proporciones (con un margen de error máximo admitido de $5 \%$ y un nivel de confianza de 99\%). A continuación, la muestra se estratificó por campo de práctica (tabla 1). Sin embargo, se tuvo disponibilidad de más unidades muestrales y en total se incluyeron 113 estudiantes. Se evaluaron 84 mujeres que equivalen al $74.3 \%$ de la muestra y 29 hombres $(25,7 \%)$. Los individuos tenían entre 19 y 65 años, con un promedio de 24 años. La frecuencia de edad entre los 19 y 24 años fue la mayor (70,8\%). Los campos de práctica más comunes entre los evaluados fueron el educativo $(36,6 \%)$ y el organizacional 
(30.1\%). El nivel de práctica que más se evidencia en la investigación es el primero, y el semestre que predominó entre los evaluados fue el sexto. Las frecuencias se observan en la tabla 2.

Tabla 1: Estratificación de la muestra

\begin{tabular}{clccc}
\hline Estrato & $\begin{array}{c}\text { Campo de } \\
\text { práctica }\end{array}$ & $\begin{array}{c}\text { Número de sujetos } \\
\text { en el estrato }\end{array}$ & Proporción & Muestra del estrato \\
\hline 1 & Clínico & 2 & $1,8 \%$ & 2 \\
2 & Deportivo & 4 & $3,5 \%$ & 3 \\
3 & Educativo & 41 & $36,3 \%$ & 35 \\
4 & Investigación & 10 & $8,8 \%$ & 9 \\
5 & Organizacional & 37 & $32,7 \%$ & 32 \\
6 & Social & 19 & $16,8 \%$ & 16 \\
& & Total & $100 \%$ & 97 \\
\hline
\end{tabular}

Tabla 2: Frecuencia para las variables demográficas

\begin{tabular}{lcc}
\hline & Frecuencia & Porcentaje \\
\hline Sexo & & \\
Mujer & 84 & 74,3 \\
Hombre & 29 & 25,7 \\
Total & 113 & 100 \\
\hline Edad & & \\
19-24 años & 80 & 70,8 \\
$25-30$ años & 22 & 19,5 \\
$31-36$ años & 7 & 6,2 \\
37-42 años & 3 & 2,7 \\
$43-65$ años & 1 &, 9 \\
Total & 113 & 100 \\
\hline Campo de práctica & & \\
Educativo & 41 & 36,3 \\
Clínico & 4 & 3,5 \\
Organizacional & 34 & 30,1 \\
Deportivo & 4 & 3,5 \\
Investigación & 11 & 9,7 \\
Social & 19 & 16,8 \\
Total & 113 & 100 \\
\hline
\end{tabular}




\begin{tabular}{lcc}
\hline & Frecuencia & Porcentaje \\
\hline Nivel de práctica & & \\
Práctica 1 & 48 & 42,5 \\
Práctica 2 & 5 & 4,4 \\
Práctica 3 & 3 & 2,7 \\
Práctica 4 & 24 & 21,2 \\
Práctica 1 y 2 & 1 &, 9 \\
Práctica 2 y 3 & 28 & 24,8 \\
Práctica 3 y 4 & 4 & 3,5 \\
Total & 113 & 100 \\
\hline Semestre en curso & & \\
6 & 41 & 36,3 \\
7 & 23 & 20,4 \\
8 & 35 & 31,0 \\
9 & 14 & 12,4 \\
Total & 113 & 100 \\
\hline
\end{tabular}

Instrumento: Se aplicó la validación española del Autoinforme de actitudes y valores en las interacciones sociales en general (ADCA-1). Consta de 35 ítems que evalúan autoasertividad (primeros 20 ítems) y heteroasertividad (15 ítems restantes), permitiendo clasificar los evaluados según su perfil de interacción, como pasivos, agresivos, asertivos y pasivo-agresivos. Se califica de forma manual, y las puntuaciones directas se transforman en centiles. Los coeficientes de correlación test-retest de la versión original son de 0,87 para autoasertividad y de 0,83 para heteroasertividad, y los índices de consistencia interna son de 0,90 para autoasertividad y de 0,85 para heteroasertividad (García \& Magaz, 2000). En el presente estudio, se obtuvo un Alfa de Cronbach de 0,915 para el total de la escala, cifra que se interpreta como un nivel de consistencia interna elevada de los resultados derivados.

Procedimiento: Se informó a la Dirección del Programa de Psicología del objetivo del estudio y se solicitó su autorización para acceder a los practicantes en las aulas de clase. Se obtuvo el consentimiento informado por escrito y, por último, se invitó a los estudiantes a participar de forma voluntaria en la investigación, garantizándoseles la confidencialidad de la información. La administración del instrumento fue realizada por la investigadora principal y duró aproximadamente 15 minutos.

Análisis de datos: Los datos se analizaron con el programa estadístico Statistical Package for the Social Sciences (SPSS) versión 24. Se hicieron análisis de 
frecuencias para las variables sociodemográficas, y se calcularon estadísticos descriptivos para las variables de estudio. Asimismo, se calculó la consistencia interna de los resultados mediante Alfa de Cronbach, se hicieron análisis intraclase, y se puso a prueba el supuesto de normalidad de las variables mediante la prueba de Kolmogorov Smirnov, y para las variables con distribución no normal se usó U de Mann-Whitney y Kruskal Wallis, finalmente se compararon las medias para las variables de estudio.

Consideraciones éticas: Se establecieron las acciones necesarias para velar por la integridad, los derechos y el bienestar de los estudiantes evaluados. Además, se guardó el rigor ético y metodológico en el uso del material psicotécnico, se presentaron de manera clara los objetivos de la investigación, y la participación de las personas fue voluntaria, previa firma de

\section{Resultados}

En la tabla 3 se puede evidenciar que las 113 personas evaluadas obtuvieron un puntaje alto en ambas variables de estudio, es decir, los individuos evaluados tienden a ser asertivos. Hay que mencionar, además, que uno de los objetivos de la investigación era comparar los niveles de autoasertividad y heteroasertividad, y que la tabla permite deducir que los practicantes profesionales que participaron en la investigación son más heteroasertivos que autoasertivos.

Tabla 3: Estadísticos descriptivos para las variables de estudio

\begin{tabular}{lccccccc}
\hline & N & Mínimo & Máximo & Media & $\begin{array}{c}\text { Desviación } \\
\text { estándar }\end{array}$ & Mediana & Rango \\
\hline Autoasertividad & 113 & 5 & 95 & 60,75 & 32,142 & 70 & 90 \\
Heteroasertividad & 113 & 5 & 95 & 67,04 & 30,703 & 80 & 90 \\
\hline
\end{tabular}

Además, con base en los resultados de la prueba de normalidad de Kolmogorov Smirnov, se estableció que las variables dependientes no tienen una distribución normal (tabla 4).

Por último, en las pruebas no paramétricas no se encontraron diferencias significativas para las variables dependientes según el sexo, la edad, el semestre en curso, el campo de práctica y el nivel de práctica. 
Tabla 4: Resultados prueba de normalidad de Kolmogorov Smirnov y pruebas no paramétricas

\begin{tabular}{lcc}
\hline \multicolumn{3}{c}{ Prueba de normalidad de Kolmogorov Smirnov } \\
& Estadístico & Sig. \\
\hline Autoasertividad &, 173 &, 000 \\
Heteroasertividad &, 215 &, 000 \\
\hline
\end{tabular}

Prueba U de Mann- Whitney

Sexo

Sig.

Autoasertividad

Heteroasertividad , 107

Prueba Kruskal-Wallis

Campo de práctica

Sig.

Autoasertividad

Heteroasertividad

Nivel de práctica

Autoasertividad

Heteroasertividad

Semestre en curso

Autoasertividad

Heteroasertividad

\section{Edad}

Autoasertividad

* Los puntajes centiles son constantes cuando Nivel de práctica $=$ Práctica 1 y 2 , y Edad $=$ 43-65 años se han omitido.

\section{Discusión}

Se evaluó el constructo de asertividad operacionalizado por medio de dos subvariables de estudio, autoasertividad y heteroasertividad. Los resultados de dichas subvariables se relacionaron con las variables demográficas (sexo, edad, ámbito de práctica, nivel de práctica, y semestre en curso). Se considera importante indicar que la confiabilidad de los resultados según el Alfa de Cronbach fue elevada $(0,915)$.

Con respecto al total de evaluados, el análisis descriptivo de la tabla 3 muestra que los 113 estudiantes obtuvieron puntajes altos en las dos variables de estudio, esto quiere decir que las unidades muestrales se caracterizan porque 
tienden a ser asertivas. Desglosando esta información, con base en los estadísticos descriptivos, el promedio de los evaluados en la variable heteroasertividad fue mayor que en autoasertividad, de lo que se puede afirmar que teniendo en cuenta que los evaluados están matriculados en una carrera de las ciencias humanas y sociales, es un resultado esperado.

Lo anterior está en línea con la propuesta de García y Magaz (2000), quienes sostienen que la heteroasertividad es definida como una conducta que respeta la expresión cordial y sincera de las emociones, valores y preferencias de otros. En consecuencia, los estudiantes de psicología de la IES deberían mostrar mayor heteroasertividad que autoasertividad, tal como se evidenció en los resultados descritos.

Por otra parte, para la variable sexo, se concluyó que no hay diferencias significativas entre los hombres y las mujeres tanto en autoasertividad como en heteroasertividad. Sin embargo, de los 113 evaluados, 84 fueron mujeres y 29 hombres, ya que la muestra no fue emparejada. De ahí que, este resultado se relacione con los hallazgos de Gutiérrez y López (2015), quienes encontraron que los hombres obtuvieron mayor puntaje que las mujeres en ambas dimensiones del ADCA, instrumento aplicado en su investigación. Adicionalmente, se debe tener en cuenta que la psicología profesional en Colombia tiene una tendencia a una mayor representación de las mujeres en el gremio, de acuerdo con cifras reportadas por el Colpsic (2012).

Por otro lado, respecto a la edad, la muestra tuvo gran dispersión, pues las edades se distribuyeron desde los 19 hasta los 65 años. Se observó una tendencia a un incremento de la asertividad en relación con la edad (a mayor edad, mayor tendencia asertiva). Lo anterior, fue corroborado por Cañón y Rodríguez (2011), cuyos resultados sugieren que a medida que aumenta la edad, se evidencian índices de asertividad más altos.

En cuanto al ámbito de práctica, se encontró que los evaluados en los campos deportivo y clínico son más asertivos, no obstante, este resultado está directamente relacionado con la frecuencia, es decir, estos son los campos en los que hubo menos participantes. Respecto a las variables de estudio, los participantes que se encuentran realizando práctica profesional en el ámbito social son los más bajos en autoasertividad y los del campo educativo son los que puntuaron con menor cifra en heteroasertividad.

En consecuencia, de acuerdo con el perfil del practicante en el ámbito social, es esperable un mayor índice de heteroasertividad, que les permita ser empáticos en el contexto de sus labores. Para el caso del practicante en el ámbito educativo, se esperarían puntuaciones similares en ambas subvaria- 
bles, que le posibiliten la justa medida en su proceder, en relación con los diferentes actores de la comunidad educativa. A pesar de ello, no fue posible encontrar antecedentes de investigación que permitan clarificar y concluir apropiadamente, a partir de los resultados descritos.

En cuanto al nivel de práctica, los practicantes más asertivos fueron los que tienen matriculados en conjunto los niveles 1 y 2 . No obstante, aquí también los resultados se ven directamente afectados por la frecuencia, ya que en este nivel de práctica solo se evidenció un participante. Los estudiantes que matricularon práctica profesional en los niveles 3 , y 2 y 3 conjuntamente, son los más hetero y autoasertivos.

Además, los estudiantes que mejor puntuaron tanto en heteroasertividad como en autoasertividad son los matriculados en los semestres séptimo y noveno, y los estudiantes del sexto semestre son los menos posicionados en ambas variables de estudio, es decir, son los menos asertivos. A propósito de esto, la investigación de León et al. (2009) en estudiantes de la facultad de ciencias de la salud, en la que se evaluaron, entre otros, estudiantes de psicología, reporta un promedio de edad que coincide con el promedio de edad del sexto semestre de la presente investigación, lo que permite deducir, tanto en el trabajo citado como en la investigación actual, que las habilidades asertivas de los evaluados no están suficientemente desarrolladas, por lo cual se evidencia una necesidad de catalizar en ellos las habilidades sociales.

En suma, los resultados de la investigación son positivos, teniendo en cuenta las características y competencias de los psicólogos profesionales, el perfil de los practicantes de psicología, y el perfil profesional de los graduados de la IES (Centro Progresa, 2017; Colpsic, 2014; Corporación Universitaria Minuto de Dios, 2015).

En contraste, los resultados obtenidos en este estudio están sujetos a diferentes limitaciones. La principal es que el instrumento que se usó para la recolección de datos no está validado en el país y por ende en el contexto, de ahí que una de las mayores recomendaciones sea que las próximas investigaciones se orienten a la adaptación y validación del ADCA en Colombia.

Otra limitación, es que la muestra no fue emparejada, esto dificultó el análisis de algunos de los datos. Si el interés de futuros investigadores es saber, en relación con el sexo, quién es más asertivo, se recomienda estructurar el estudio con una muestra pareja.

Adicionalmente, considerando que, al momento de realizar el rastreo de antecedentes de investigación, se encontraron pocos estudios sobre asertividad 
en practicantes, se recomienda proponer más investigaciones sobre habilidades sociales y asertividad en la población evaluada.

Se recomienda también, ampliar la presente investigación, con marcos muestrales mayores, que posibiliten aseveraciones y generalizaciones con mayor poder estadístico, teniendo en cuenta que la cantidad de estudiantes de psicología de las IES aumenta cada semestre de forma considerable.

Finalmente, se propone realizar investigaciones a nivel local, regional, y nacional, sobre el nivel de asertividad de los estudiantes universitarios de psicología, con el fin de comparar e intervenir de manera más objetiva los posibles aspectos a mejorar.

\section{Referencias}

Behar, R., Manzo, R., Casanova, D. (2006). Trastornos de la conducta alimentaria y asertividad. Revista Médica de Chile, 134 (3): 312-319. Recuperado de http:// www.scielo.cl/pdf/rmc/v134n3/art07.pdf

Caballo, V. (1983). Asertividad: definiciones y dimensiones. Estudios de Psicología, 13: 52-62. Recuperado de https://dialnet.unirioja.es/servlet/articulo? codigo $=65876$

Caballo, V. (1986). Manual de evaluación de entrenamiento y habilidades sociales. España: Siglo Veintiuno Editores.

Cañón, W., Rodríguez, A.L. (2011). Asertividad: una habilidad social necesaria en los profesionales de enfermería y fisioterapia. Texto \& Contexto enfermagem, 20: 8187. Recuperado de http://www.redalyc.org/articulo.oa?id=71421163010

Centro Progresa (2017). Perfil practicante de Psicología. Corporación Universitaria Minuto de Dios: Bello.

Colegio Colombiano de Psicólogos (2012). Condiciones sociodemográficas, educativas, laborales y salariales del psicólogo colombiano. Bogotá: Autor. Recuperado de https://issuu.com/colpsic/docs/condiciones_del_psicologo/6

Colegio Colombiano de Psicólogos (2014). Perfil y competencias del Psicólogo en Colombia, en el contexto de la salud. Bogotá: Autor. Recuperado de https://www. minsalud.gov.co/sites/rid/Lists/BibliotecaDigital/RIDE/VS/TH/Psicologia_ Octubre2014.pdf

Coppari, N., Aponte, N., Bauzá, H., Corina, C., Dancuart, N., García, A., ... Smiliansky, I. (2009). Conducta asertiva y autoeficacia para la prevención de ETS en jóvenes estudiantes de la ciudad de asunción. Eureka, 6 (1): 7-16. Recuperado de http:// www.psicoeureka.com.py/sites/default/files/articulos/eureka-6-1-09-6.pdf 
Corporación Universitaria Minuto de Dios (2015). Proyecto Curricular del Programa. Psicología. Bello: Autor.

García, E.M., Magaz, A. (2000). Autoinformes de Actitudes y Valores en las Interacciones Sociales: ADCAs. Recuperado de https://gac.com.es/editorial/INFO/Manuales/ adcaMANU.pdf

Gismero, E. (2000). EHS: Escala de Habilidades Sociales. Madrid: TEA Ediciones.

González, C., Guevara, Y., Jiménez, D., Alcázar, R.J. (2018). Relación entre asertividad, rendimiento académico y ansiedad en una muestra de estudiantes mexicanos de secundaria. Acta Colombiana de Psicología, 21 (1): 116-127. doi: 10.14718/ACP.2017.21.1.6

Gutiérrez, M., López, J. (2015). Autoconcepto, dificultades interpersonales, habilidades sociales y conductas asertivas en adolescentes. Revista Española de Orientación y Psicopedagogía, 26 (2): 42-58. Recuperado de http://www.redalyc. org/articulo.oa?id=338241632004

Kelly, J. (2002). Entrenamiento de las habilidades sociales. Bilbao: Desclée de Brouwer.

León, A., Rodríguez, C., Ferrel, F.R., Ceballos, G.A. (2009). Asertividad y autoestima en estudiantes de primer semestre de la Facultad de Ciencias de la Salud de una universidad pública de la ciudad de Santa Marta (Colombia). Psicología desde el Caribe, 24: 91-105. Recuperado de http://www.scielo.org.co/pdf/psdc/n24/ n24a05.pdf

Londoño, C., Valencia, C. (2008). Asertividad, resistencia a la presión de grupo y consumo de alcohol en universitarios. Acta colombiana de psicología, 11 (1): 155162. Recuperado de http://www.scielo.org.co/pdf/acp/v11n1/v11n1a16.pdf

Llacuna, J., Pujol, L. (2004). NTP 667: La conducta asertiva como habilidad social. España: Ministerio de Trabajo y Asuntos Sociales. Recuperado de http:// www.insht.es/InshtWeb/Contenidos/Documentacion/FichasTecnicas/NTP/ Ficheros/601a700/ntp_667.pdf

Merino, M.I. (2010). Habilidades sociales. En Manual para formadores de voluntariado (pp.68 - 79). Castilla - La Mancha, España: Consejería de Salud y Bienestar Social Junta de Comunidades de Castilla-La Mancha. Recuperado de https://dialnet. ezproxy.uniminuto.edu/servlet/articulo?codigo $=3732137$

Roca, E. (2003). Cómo mejorar tus habilidades sociales (4 ${ }^{\mathrm{a}}$ Ed. Rev.). Valencia: ACDE Ediciones. Recuperado de https://www.cop.es/colegiados/PV00520/pdf/ Habilidades\%20sociales-Dale\%20una\%20mirada.pdf

Rondón, J.E., Cardozo, I., Lacasella, R. (2018). Influencia de la depresión, los estilos de comunicación y la adhesión al tratamiento sobre los niveles de glucosa 
en personas con diabetes. Acta Colombiana de Psicología, 21 (2): 39-53. doi: 10.14718/ACP.2018.21.2.3

Torres, L.E. (2015). Asertividad en practicantes de Psicología de la Universidad de Antioquia Sede Amalfi (Tesis de pregrado). Universidad de Antioquia, Amalfi, Colombia. Recuperado dehttp://200.24.17.74:8080/jspui/bitstream/fcsh/357/1/ TorresLuz_asertividadpracticantespsicologiauniversidadantioquiaamalfi.pdf

Velázquez, M., Arellanez, J., Martínez, A. (2012). Asertividad y consumo de drogas en estudiantes mexicanos. Acta Colombiana de Psicología, 15 (1): 131-141. Recuperado de https://editorial.ucatolica.edu.co/ojsucatolica/revistas_ucatolica/ index.php/acta-colombiana-psicologia/article/view/203/244 